Check for updates

Cite this: Mater. Adv., 2020 1,1125

Received 18th April 2020, Accepted 19th June 2020

DOI: 10.1039/d0ma00220h

rsc.li/materials-advances

\title{
Green synthesis of air-stable tellurium nanowires via biomolecule-assisted hydrothermal for thermoelectrics $\dagger$
}

\author{
Youfa Liu, ${ }^{a}$ Qinglin Jiang, ${ }^{* b}$ Jiang Zhang, ${ }^{b}$ Wenjun Ding, ${ }^{a}$ Jingkun $X u$, (D) *ac \\ Peipei Liu, ${ }^{a}$ Guangming Nie, (D) c Haolai Mao ${ }^{a}$ and Fengxing Jiang iD *a
}

\begin{abstract}
Tellurium nanowires (Te NWs) have been widely investigated in energy generators, photoelectric devices, sensors and chemical templates due to their interesting properties. However, these promising applications can be critically hindered by the inferior electrical conductivity of Te NWs and the ungreen and high energy consumption of the synthesis process. Herein, we suggest a feasible and eco-friendly strategy to synthesize Te NWs via a hydrothermal process by employing the biomolecule glucose as the reductant. The suggested strategy not only successfully avoided the use of toxic and harsh reductants and additional surfactants but also obtained Te NWs, even at low reaction temperatures. Moreover, the synthesized Te NWs presented a high electrical conductivity of $8.44 \mathrm{~S} \mathrm{~cm}^{-1}$ by optimizing the reaction conditions, and a large power factor of $97.3 \mu \mathrm{W} \mathrm{m} \mathrm{m}^{-1} \mathrm{~K}^{-2}$ can be obtained after annealing. Combining the variable range hopping $(\mathrm{VRH})$ model with the carrier transport parameters, the PVP surfactant-weakened carrier transporting mechanism was proposed. With the as-assembled thermoelectric generator, a promising output voltage of $231 \mathrm{mV}$ can be realized when the temperature difference is $80 \mathrm{~K}$. The strategy shows a novel design for synthesizing highly conductive Te NWs in an eco-friendly process and presents a promising Te NWs-based thermoelectric material.
\end{abstract}

\section{Introduction}

Tellurium (Te), one of the promising members of the chalcogen family, ${ }^{1,2}$ has been intensively investigated in the applications of optoelectronics, ${ }^{3,4}$ piezoelectric devices, ${ }^{5}$ gas sensors, ${ }^{6}$ thermoelectric generators (TEGs) $)^{7,8}$ and chemical templates ${ }^{9,10}$ because of its interesting properties. Among these applications, TEGs have obtained considerable attention due to the properties of no moving parts, no emissions, long life, being light-weight, as well as the capability of converting electric energy from waste heat, ${ }^{11,12}$ and is expected to be applied in the field of green energy conversion in the future. Generally, the energy converting efficiency of TEGs can be defined as a dimensionless parameter, the figure-of-merit (ZT): $Z T=\sigma S^{2} T / \kappa$, where $\sigma, S, \kappa$ are the electrical conductivity, Seebeck coefficient and thermal conductivity of the

\footnotetext{
${ }^{a}$ Flexible Electronics Innovation Institute, Jiangxi Science and Technology Normal University, Nanchang 330013, P. R. China. E-mail: xujingkun1971@yeah.net, f.x.jiang@live.cn

${ }^{b}$ Institute of Polymer Optoelectronic Materials and Devices, State Key Laboratory of Luminescent Materials and Devices, South China University of Technology, Guangzhou 510640, P. R. China. E-mail: jiangql@scut.edu.cn

${ }^{c}$ School of Chemistry and Molecular Engineering, Qingdao University of Science and Technology, Qingdao 266042, Shandong, P. R. China

$\dagger$ Electronic supplementary information (ESI) available. See DOI: 10.1039/ d0ma00220h
}

TE material, respectively, and $T$ is the absolute temperature. ${ }^{13,14} \mathrm{As}$ a good TE material, high electrical conductivity, large Seebeck coefficient, and low thermal conductivity are highly desired to realize the high $Z T$ value. However, the inferior electrical conductivity $\left(0.005-0.15 \mathrm{~S} \mathrm{~cm}^{-1}\right)^{15,16}$ of the current Te nanomaterials (Te NMs) has critically hindered its applications in TEG and others.

To realize the novel applications of Te NMs, a simple and feasible strategy for synthesizing Te NMs with a controllable and uniform topography in a green method is urgently required. ${ }^{1,17}$ To date, various morphologies, such as nanowires, ${ }^{18}$ nanorods, ${ }^{19}$ nanotubes, ${ }^{20}$ nanoflakes, ${ }^{21}$ and even tellurene,${ }^{22}$ of Te NMs have been investigated, and the corresponding growth mechanisms have also been developed. For example, the solid-solution-solid growth mechanism and the nucleation-dissolution-recrystallization growth mechanism have been proposed for the generation of $\mathrm{Te} \mathrm{NWs}^{23}$ and Te nanotubes. ${ }^{24}$ Furthermore, the intrinsically crystallography-dependent topography has also been developed, even in the absence of surfactants. ${ }^{25}$ However, most of the currently developed methods for synthesizing Te NMs are based on wet chemistry processes, where toxic reductants such as hydrazine hydrate ${ }^{26}$ or sodium borohydride ${ }^{27}$ are involved. To avoid the harms from such reductants to either humans or the environment, other mild or non-toxic reductants (ethylene glycol, ascorbic acid) have been widely utilized. ${ }^{28,29}$ Since the initially generated $\mathrm{Te}$ atoms in wet chemistry processes, which 
are so-called seeds in the reaction medium, generally tend to aggregate due to their high free energy, it is hard to present a specific topography of Te NMs under such conditions. ${ }^{30}$ Fortunately, this issue can be solved by employing surfactants such as polyvinylpyrrolidone (PVP), ${ }^{31}$ sodium dodecylbenzenesulfonate (SDBS), ${ }^{32}$ and cetyltrimethylammonium bromide $(\mathrm{CTAB})^{33}$ as both stabilizers and templates.

Surfactants are usually employed to stabilize and induce the topography of Te NMs by adsorbing on the surface of Te NMs. ${ }^{34,35}$ However, the adsorption between surfactants and Te NMs may inevitably block the contacts between Te NMs, resulting in inferior electrical conductivity to Te NMs. ${ }^{15}$ It is also a challenge to remove the surfactants from the surface of Te NMs without undesired effects. In addition, the currently developed strategies for synthesizing Te NMs based on wet chemistry usually require high temperatures $\left(170-200{ }^{\circ} \mathrm{C}\right),{ }^{21,36}$ and so are risk- and energyconsuming. Recently, bacteria-assisted strategies have also been developed to synthesize Te NMs, where the bacterium is selected as the Te NMs generator in a tellurite medium. ${ }^{37,38}$ Compared with wet chemistry, this strategy successfully avoids the use of harsh reagents (toxic reductants) and surfactants, and it is safer without the additional energy input. However, it would be obstructed by the harsh survival environment of bacillus, where both the $\mathrm{pH}$ value and temperature of the medium would dramatically affect the survival of the bacterium. It is also time-consuming and not suitable for scale-up.

Glucose, one of the eco-friendly biomolecules, has been widely utilized to synthesize metal nanomaterials because of its reducing properties. Metal or non-metal nanomaterials, e.g., $\mathrm{Au}, \mathrm{Ag}, \mathrm{Cu}, \mathrm{Pt}$ and Se, have been successfully synthesized by the hydrothermal process involving glucose as a reductant. ${ }^{39-41}$ On the other hand, carbonaceous nanotubes or carbonaceous Ag (Ag@C core-shell) nanocables have also been fabricated by carbonizing glucose on the surface of Te NWs or Ag NWs, and these materials can be applied in diverse fields. ${ }^{42-44}$ However, the investigation of the synthesis of Te NMs with glucose is very rare, ${ }^{45}$ and the TE performance of Te NMs obtained in such a way have also never been studied. Compared with the abovementioned strategies, glucose would be one of the most promising candidates for synthesizing Te NMs due to the following advantages. (1) Glucose is a green reductant, eco-friendly and bio-friendly. (2) It is renewable, easy-to-get and low-cost. (3) Apart from acting as a reductant, glucose can also act as a surfactant in the hydrothermal process. (4) Due to the good water-solubility of glucose, the re-dispersibility of Te NMs would be considerable when glucose is employed as a reductant. (5) No high temperature is needed due to the reduction of the aldehyde group.

In this work, we developed a simple and eco-friendly strategy to synthesize Te NWs by utilizing the biomolecule glucose as the reductant. Herein, no toxic reductant and additional surfactant were involved, and the reaction conditions were also systematically investigated. With the synthesized Te NWs, thin pieces were fabricated by combining the vacuum filtration and pressing process, and their TE performances were also systematically studied by varying the reaction and annealing conditions. To understand the high electrical conductivity of the as-fabricated
Te NWs thin pieces, the one-dimensional variable range hopping (VRH) model was fitted based on the temperature-dependent electrical conductivity. Furthermore, a simple TEG with 10 pieces of the as-fabricated Te NWs thin pieces was assembled, and the output performance was measured at the temperature difference from 0 to $80 \mathrm{~K}$. Finally, the air-stable TE performance of the as-fabricated Te NWs thin piece was illustrated by investigating the time-dependence of the TE performance. This work will provide a green and feasible method for synthesizing highly conductive Te NWs and develop a new strategy to design a promising Te NWs-based TE material.

\section{Experimental}

\section{Materials}

Sodium tellurite $\left(\mathrm{Na}_{2} \mathrm{TeO}_{3}, \geq 99 \%\right)$, glucose $\left(\alpha-\mathrm{D}-(+)-\mathrm{C}_{6} \mathrm{H}_{12} \mathrm{O}_{6}, 99 \%\right)$, polyvinylpyrrolidone (PVP, $\left.M_{\mathrm{W}}=40000\right), \mathrm{NH}_{3} \cdot \mathrm{H}_{2} \mathrm{O}(25-28 \%$ ), $\mathrm{N}_{2} \mathrm{H}_{4} \cdot \mathrm{H}_{2} \mathrm{O}(80 \%)$, absolute ethanol (EtOH, 99.8\%) and acetone were all purchased from J\&K Scientific Ltd. All the materials were used as received.

\section{Methods}

\section{Synthesis of Te NWs with glucose as the reductant}

In a typical procedure for the synthesis of Te NWs, $140.0 \mathrm{mg}$ $\mathrm{Na}_{2} \mathrm{TeO}_{3}$ and $3.9872 \mathrm{~g}$ glucose were dissolved in $80 \mathrm{~mL}$ deionized water (DI) to obtain a colorless transparent solution. After this process, the resulting solution was poured into a $100 \mathrm{~mL}$ sealed reactor and reacted at $120{ }^{\circ} \mathrm{C}$ for $12 \mathrm{~h}$. The Te NWs were obtained in the reaction solution.

\section{Synthesis of Te NWs with $\mathrm{N}_{2} \mathrm{H}_{4} \cdot \mathrm{H}_{2} \mathrm{O}$ as the reductant and PVP as the surfactant}

The Te NWs with $\mathrm{N}_{2} \mathrm{H}_{4} \cdot \mathrm{H}_{2} \mathrm{O}$ as the reductant and PVP as the surfactant were synthesized as previously reported. ${ }^{46}$ Briefly, $188.4 \mathrm{mg} \mathrm{Na} \mathrm{TeO}_{3}$ and $1.000 \mathrm{~g}$ PVP were dissolved in $66 \mathrm{~mL}$ of DI under vigorous magnetic stirring at room temperature to obtain a homogeneous solution. Then, $3.3 \mathrm{~mL} \mathrm{~N} \mathrm{H}_{4} \cdot \mathrm{H}_{2} \mathrm{O}$ and $6.7 \mathrm{~mL} \mathrm{NH} \mathrm{NH}_{3} \cdot \mathrm{H}_{2} \mathrm{O}$ were added to the solution, while stirring. Finally, the solution was poured into a $100 \mathrm{~mL}$ sealed reactor and then reacted at $185{ }^{\circ} \mathrm{C}$ for $3.5 \mathrm{~h}$ to obtain the Te NWs.

\section{Fabrication of Te NWs thin pieces and TE generator}

For the TE performance test, the Te NWs thin pieces were fabricated as follows. The reaction solution was naturally cooled to room temperature and divided evenly into two. Each part was fabricated into a thin piece (diameter of $\sim 2.0 \mathrm{~cm}$ ) with the PVDF microporous membrane filter $(0.22 \mu \mathrm{m})$ as the substrate via vacuum filtration and then washed several times with DI and EtOH. The cleaned thin pieces were dried in a vacuum oven at $60{ }^{\circ} \mathrm{C}$ for $12 \mathrm{~h}$, and then a free-standing Te NWs thin piece was fabricated by peeling it from the substrate. Finally, this thin piece was pressed under $15 \mathrm{MPa}$ for $15 \mathrm{~min}$. Here, the fabrication of the Te NWs thin piece with $\mathrm{N}_{2} \mathrm{H}_{4} \cdot \mathrm{H}_{2} \mathrm{O}$ 
as the reductant was slightly different from that of glucose, which is presented in the ESI. $\dagger$

To design the TE generator, the as-fabricated Te NWs thin piece was cut into rectangles $\left(13.0 \times 2.00 \mathrm{~mm}^{2}\right)$ and 10 of the rectangles were connected in series by copper wires and silver paste (as shown in Fig. 6A). The output performance of the asfabricated TE generator was evaluated by building a temperature difference $(\Delta T)$ at the two ends of the rectangles at room temperature.

\section{Instruments}

The scanning electron microscope (SEM) images were obtained using a JSM-7500F instrument. X-ray diffraction (XRD) patterns were recorded on a DX-2700 B X-ray diffractometer. X-ray photoelectron spectra (XPS) were obtained on a Thermo ESCALAB 250Xi. The EDS spectrum was obtained on a GENESIS (EDAX Inc., USA) instrument. The carrier concentration and mobility of the as-fabricated thin pieces were obtained by an HMS-3000 Hall Tester. The electrical conductivity was measured by employing a standard four-point probe technique with a Keithley 2700 to measure the resistance $(R)$ of the sample, and the electrical conductivity was calculated as: $\sigma=L /(R \cdot A)$, where $L$ and $A$ are the length and the cross-sectional area of the sample. The Seebeck coefficient was obtained as $S=-\Delta V / \Delta T$, where $\Delta V$ is the voltage gradient between the two ends of the sample, measured by Keithley 2700 and 2401 systems (Scheme S1, $\mathrm{ESI} \dagger) . \Delta T$ is the temperature gradient at the two ends of the sample, which was built by an Ohm resistive heater.

\section{Results and discussion}

\section{Synthesis and characterization}

Fig. 1 shows the schematic illustration of the synthesis of Te NWs with the biomolecule glucose as the reductant via the hydrothermal method and the fabrication of the Te NWs thin pieces. In this work, sodium tellurite was used as the tellurium source, and glucose was

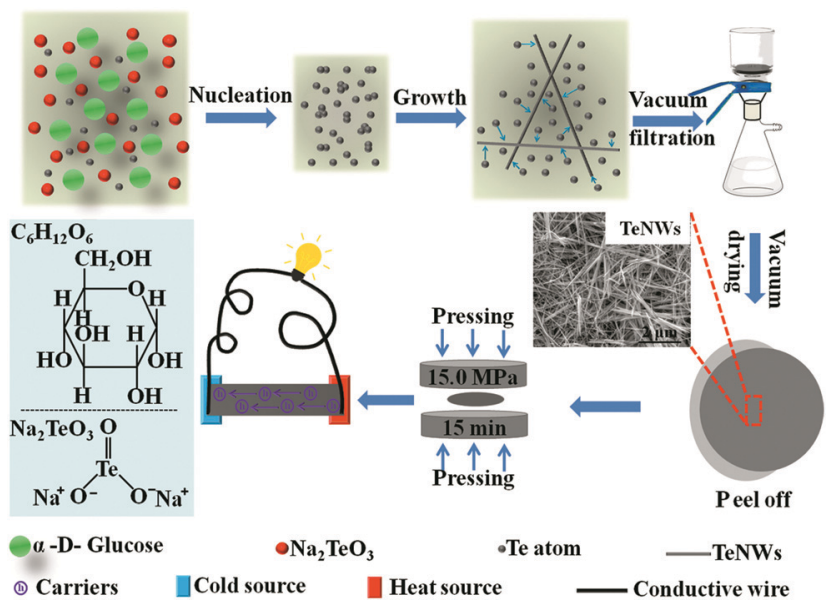

Fig. 1 Schematic illustration of the synthesis of Te NWs with glucose as the reductant, and the fabrication of Te NWs thin pieces.
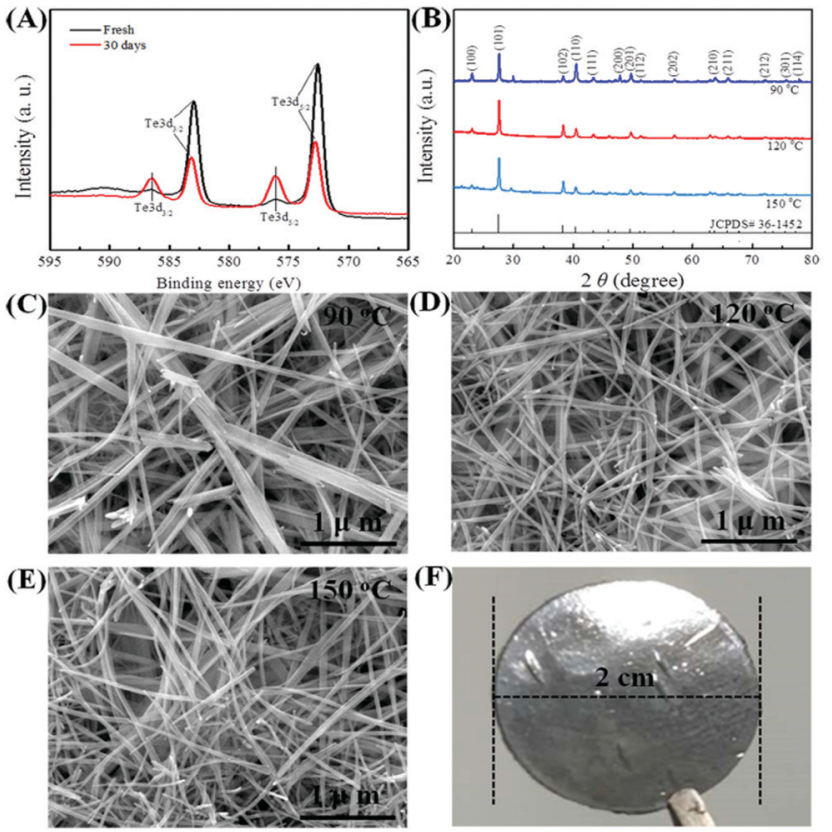

Fig. 2 Te3d XPS spectra (A) of the fresh Te NWs synthesized with glucose and those stored under ambient conditions for 30 days. XRD pattern (B) and SEM images (C-E) of the Te NWs synthesized with glucose at $90{ }^{\circ} \mathrm{C}(\mathrm{C})$, $120{ }^{\circ} \mathrm{C}$ (D), $150{ }^{\circ} \mathrm{C}$ (E) for $12 \mathrm{~h}$. Photo of the optimized Te NWs thin piece (F).

employed as both the reductant and surfactant. The reaction between $\mathrm{TeO}_{3}{ }^{2-}$ and glucose is as follows: ${ }^{47}$

$$
\begin{gathered}
2 \mathrm{CH}_{2} \mathrm{OH}-(\mathrm{CHOH})_{4}-\mathrm{CHO}+\mathrm{TeO}_{3}{ }^{2-} \rightarrow 2 \mathrm{CH}_{2} \mathrm{OH}-(\mathrm{CHOH})_{4}- \\
\mathrm{COO}^{-}+\mathrm{Te}+\mathrm{H}_{2} \mathrm{O} .
\end{gathered}
$$

XPS spectra (Fig. 2A) and XRD patterns (Fig. 2B) were obtained to verify the generation of Te NWs and their phase structure. As seen in Fig. 2A, the Te NWs synthesized with glucose at $120{ }^{\circ} \mathrm{C}$ for $12 \mathrm{~h}$ presented two typical $\mathrm{Te}_{3 / 2}$ and Te $3 \mathrm{~d}_{5 / 2}$ peaks, ${ }^{48}$ suggesting that glucose can act as the reductant to synthesize Te. To optimize the reaction conditions for synthesizing Te NWs, the reaction temperature (from 60 to $180{ }^{\circ} \mathrm{C}$ ) and reaction time (from 10 to $20 \mathrm{~h}$ ) were varied. Fig. $2 \mathrm{~B}$ shows the XRD patterns of Te NWs synthesized at 90, 120, $150{ }^{\circ} \mathrm{C}$ for $12 \mathrm{~h}$, while the Te NWs were synthesized at $180{ }^{\circ} \mathrm{C}$ for $12 \mathrm{~h}$; the $\mathrm{N}_{2} \mathrm{H}_{4} \cdot \mathrm{H}_{2} \mathrm{O}$-synthesized Te NWs are presented in Fig. S1 (ESI $\dagger$ ). As shown in Fig. 2B, the Te NWs synthesized with glucose presented identical diffraction peaks, which can be indexed to the standard card (JCPDS\# 36-1452) of tellurium with a hexagonal phase. ${ }^{31}$ The results from the XRD patterns further indicate that Te can be synthesized with glucose as the reductant, even if the hydrothermal temperature was as low as $60{ }^{\circ} \mathrm{C}$. Compared with other methods, ${ }^{49,50}$ Te can be easily synthesized in this work with no toxic and harsh reagents and no high temperature involved, suggesting that this strategy is more green and is feasible for synthesizing Te NMs.

Fig. 2C-E displays the SEM images of the Te NWs synthesized with glucose at $90{ }^{\circ} \mathrm{C}(\mathrm{C}), 120{ }^{\circ} \mathrm{C}(\mathrm{D})$ and $150{ }^{\circ} \mathrm{C}(\mathrm{E})$ for $12 \mathrm{~h}$; the Te NWs synthesized with $\mathrm{N}_{2} \mathrm{H}_{4} \cdot \mathrm{H}_{2} \mathrm{O}$ are presented in 
Fig. S2 (ESI $\dagger$ ). As can be seen, all Te NWs had a nanowire morphology, which can be ascribed to the anisotropy of Te NWs along its $c$-axis. ${ }^{16}$ Due to the strong polarity and the long chain of PVP, it can effectively control the nucleation and growth of Te NWs due to the template effect and steric effect as reported in previous works. ${ }^{32,51}$ Therefore, the Te NWs synthesized with $\mathrm{N}_{2} \mathrm{H}_{4} \cdot \mathrm{H}_{2} \mathrm{O}$ were significantly thinner and longer as compared to the glucose-synthesized Te NWs. Notably, the Te NWs can be synthesized in this work even in the absence of surfactant, which can be attributed to the surfactant effect of glucose. Similarly, there are many previous reports in which glucose was utilized as a surfactant to synthesize nanomaterials. ${ }^{52-54}$ To further illustrate that glucose can act as a surfactant in this work, C element was detected in the Te NWs synthesized with glucose at $120{ }^{\circ} \mathrm{C}$ for $12 \mathrm{~h}$ by EDS spectroscopy (Table S1, ESI $\dagger$ ), where $\mathrm{C}$ arose from glucose being adsorbed on the surface of Te NWs. Based on the dual-role of glucose, the glucose-assisted self-assembly mechanism was proposed for the synthesis of Te NWs. Firstly, sodium tellurite was reduced to the Te nuclei by glucose in the solution. After that, with the assistance of the glucose surfactant, these fresh Te nuclei would prefer to assemble on the glucose surfactant to minimize their free energy. The Te nuclei adsorbed on the glucose surfactant would act as crystal seeds to adsorb Te atoms from solution to form the larger Te nuclei. Finally, the generated Te atoms in the solution would continuously adsorb onto their vicinal mature Te nuclei and grow along its $c$-axis, due to the inherently anisotropic property of trigonal Te. Therefore, Te NWs can be generated with the consumption of Te in the solution. Fig. $2 \mathrm{~F}$ shows the photo of the as-fabricated Te NWs thin piece after pressing under $15 \mathrm{MPa}$ for $15 \mathrm{~min}$, revealing the dense and smooth property of the pressed materials.

\section{Thermoelectric properties}

To evaluate the TE performance of the as-fabricated Te NWs thin pieces, these thin pieces were cut into rectangles with a scale of $13.0 \times 2.00 \mathrm{~mm}^{2}$, and the influences of the reaction temperature (from 60 to $180{ }^{\circ} \mathrm{C}$ ) and reaction time (from 10 to $20 \mathrm{~h}$ ) on the TE performance of these as-fabricated Te NWs thin pieces were systematically investigated. Fig. 3A-C presents the TE performance of these as-fabricated Te NWs thin pieces, where these Te NWs were synthesized with glucose at $60{ }^{\circ} \mathrm{C}(\mathrm{A})$, $90{ }^{\circ} \mathrm{C}(\mathrm{B}), 120{ }^{\circ} \mathrm{C}$ (C) for different reaction times. The TE performances of the Te NWs synthesized with glucose at 150 and $180{ }^{\circ} \mathrm{C}$ were not presented here due to their much too low electrical conductivity. As seen in Fig. 3A-C, the TE performance of these as-fabricated Te NWs thin pieces were not only dependent on the reaction time, but were also dependent on the reaction temperature. Fig. 3D displays the optimized TE performances of these as-fabricated Te NWs thin pieces, where the Te NWs were synthesized with glucose at different reaction temperatures. For comparison, the TE performance of the $\mathrm{N}_{2} \mathrm{H}_{4} \cdot \mathrm{H}_{2} \mathrm{O}$-synthesized Te NWs is also displayed. As observed in Fig. 3D, the optimized electrical conductivity was enhanced with the increase in the reaction temperature. A maximum electrical conductivity of $8.44 \mathrm{~S} \mathrm{~cm}^{-1}$ was obtained for Te NWs
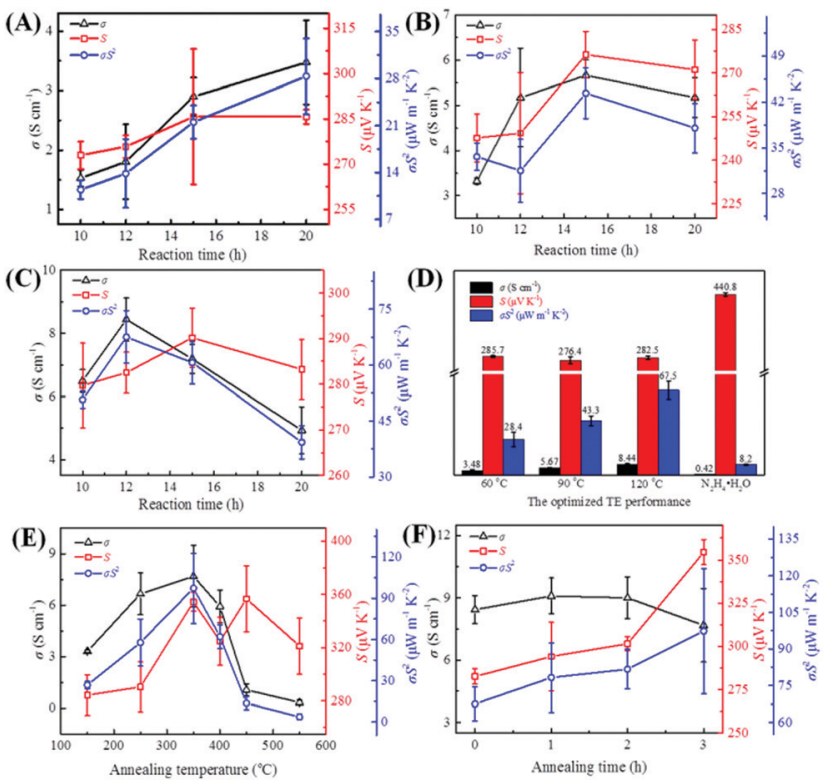

Fig. 3 Reaction condition-dependent TE performance of the Te NWs thin pieces, where the Te NWs were synthesized with glucose at $60{ }^{\circ} \mathrm{C}(\mathrm{A})$, $90{ }^{\circ} \mathrm{C}(\mathrm{B}), 120{ }^{\circ} \mathrm{C}$ (C) for different times. A comparison of the optimized TE performance of the Te NWs thin pieces (D). The TE performance of the optimized Te NWs thin pieces as a function of annealing temperature (E) and time (F).

synthesized with glucose at $120{ }^{\circ} \mathrm{C}$ for $12 \mathrm{~h}$, which is 20 times higher than that of the $\mathrm{N}_{2} \mathrm{H}_{4} \cdot \mathrm{H}_{2} \mathrm{O}$-synthesized Te NWs $\left(0.42 \mathrm{~S} \mathrm{~cm}^{-1}\right)$ in our work. Furthermore, the electrical conductivities of these as-fabricated Te NWs thin pieces in this work are 1 to 3 orders of magnitude higher as compared to previously reported works $\left(0.005-0.22 \mathrm{~S} \mathrm{~cm}^{-1}\right) .{ }^{15,55-58}$ On the other hand, as observed in Fig. 3A-D, the $S$ of the Te NWs thin pieces synthesized with glucose changed slightly under different reaction conditions. An optimized value of $285.7 \mu \mathrm{V} \mathrm{K}^{-1}$ was obtained when the reaction temperature was kept at $60{ }^{\circ} \mathrm{C}$. The $S$ value of the $\mathrm{N}_{2} \mathrm{H}_{4} \cdot \mathrm{H}_{2} \mathrm{O}$-synthesized Te NWs in this work is $440 \mu \mathrm{V} \mathrm{K}^{-1}$, which is consistent with Park's work. ${ }^{34}$ In terms of the power factor $\left(\sigma S^{2}\right)$, the optimized $\sigma S^{2}$ value was enhanced with the increase in the reaction temperature (Fig. 3D). A value of $67.5 \mu \mathrm{W} \mathrm{m} \mathrm{m}^{-1} \mathrm{~K}^{-2}$ was realized due to the high electrical conductivity of the optimized Te NWs thin pieces, where the Te NWs were synthesized with glucose at $120{ }^{\circ} \mathrm{C}$ for $12 \mathrm{~h}$. As listed in Table 1 , the $\sigma S^{2}$ for the glucose-synthesized Te NWs was not only significantly higher than that synthesized with $\mathrm{N}_{2} \mathrm{H}_{4} \cdot \mathrm{H}_{2} \mathrm{O}$ $\left(8.2 \mu \mathrm{W} \mathrm{m}{ }^{-1} \mathrm{~K}^{-2}\right)$ but was also 1 to 2 orders of magnitude larger than that previously reported, which is more promising for energy conversion.

To further improve the TE performance of the optimized Te NWs thin pieces, they were annealed under different conditions. Fig. $3 \mathrm{E}$ and $\mathrm{F}$ shows the TE performance of these optimized Te NWs thin pieces after annealing; the TE performance of the $\mathrm{N}_{2} \mathrm{H}_{4} \cdot \mathrm{H}_{2} \mathrm{O}$-synthesized Te NWs after annealing are presented in Fig. S3 (ESI $\dagger$ ). As observed in Fig. 3E, the electrical conductivity of the optimized Te NWs thin pieces increased with the increasing annealing temperature, but a further increase in temperature 
Table 1 Comparison of carrier transport parameters and TE performance for Te NMs

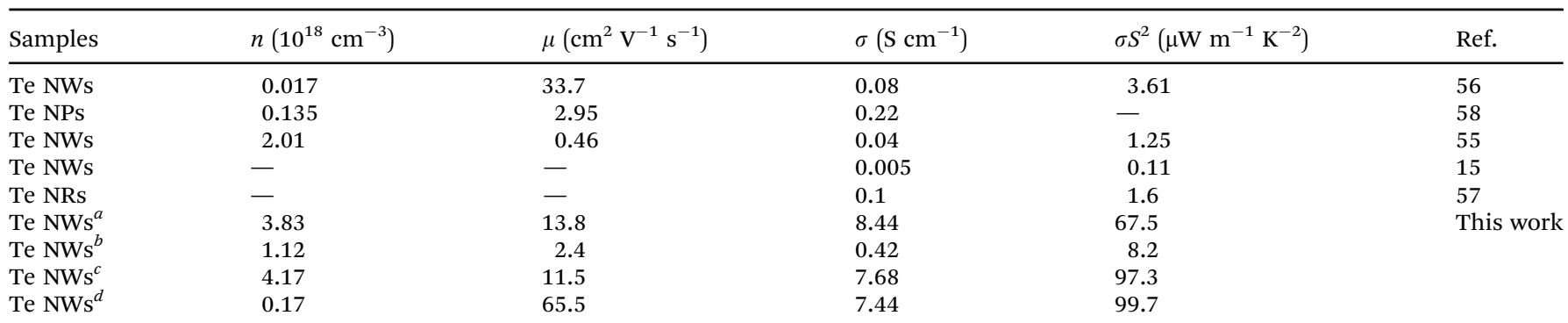

${ }^{a}$ The Te NWs were synthesized with glucose at $120{ }^{\circ} \mathrm{C}$ for $12 \mathrm{~h}$ and without annealing. ${ }^{b}$ The Te NWs were synthesized with $\mathrm{N}_{2} \mathrm{H}_{4} \cdot \mathrm{H}_{2} \mathrm{O}$ and without annealing. ${ }^{c}$ The Te NWs were synthesized with glucose at $120^{\circ} \mathrm{C}$ for $12 \mathrm{~h}$ and this as-fabricated Te NWs thin piece was annealing at $350{ }^{\circ} \mathrm{C}$ for $3 \mathrm{~h}$. ${ }^{d}$ The Te NWs were synthesized with $\mathrm{N}_{2} \mathrm{H}_{4} \cdot \mathrm{H}_{2} \mathrm{O}$ and this as-fabricated Te NWs thin piece was annealing at $350{ }^{\circ} \mathrm{C}$ for $3 \mathrm{~h}$.

led to decreased electrical conductivity. However, the maximum electrical conductivity $\left(7.68 \mathrm{~S} \mathrm{~cm}^{-1}\right)$ of the annealed Te NWs thin pieces was lower than that of the unannealed pieces $\left(8.44 \mathrm{~S} \mathrm{~cm}^{-1}\right)$, which may result from the thin pieces being relatively less dense after annealing, where the density of the optimized Te NWs thin pieces decreased from 2.4 to $2.2 \mathrm{~g} \mathrm{~cm}^{-3}$ after annealing. The $S$ value of the annealed Te NWs thin pieces was improved to $356.8 \mu \mathrm{V} \mathrm{K}^{-1}$ after annealing at $450{ }^{\circ} \mathrm{C}$ for $3 \mathrm{~h}$, and an enhanced power factor of $97.3 \mu \mathrm{W} \mathrm{m}{ }^{-1} \mathrm{~K}^{-2}$ was achieved on annealing at $350{ }^{\circ} \mathrm{C}$ for $3 \mathrm{~h}$. The enhanced value is significantly higher as compared to the previous works as listed in Table 1 . In addition, we varied the annealing time from 0 to $3 \mathrm{~h}$ at the optimized annealing temperature of $350{ }^{\circ} \mathrm{C}$. As shown in Fig. 3F, the electrical conductivity was slightly increased and then decreased with increasing annealing time. The Seebeck coefficient was increased monotonically, and the maximum power factor was reached at $350{ }^{\circ} \mathrm{C}$ for $3 \mathrm{~h}$. It is worth noting that the $\mathrm{TE}$ performance of the Te NWs synthesized with $\mathrm{N}_{2} \mathrm{H}_{4} \cdot \mathrm{H}_{2} \mathrm{O}$ was greatly enhanced due to the improved electrical conductivity (7.44 $\mathrm{S} \mathrm{cm}^{-1}$ ) in Fig. S3 (ESI $\dagger$ ), and a large power factor of $99.7 \mu \mathrm{W} \mathrm{m}{ }^{-1} \mathrm{~K}^{-2}$ was obtained on annealing at $350{ }^{\circ} \mathrm{C}$ for $3 \mathrm{~h}$. According to the previous work, ${ }^{56}$ this greatly improved electrical conductivity can be ascribed to the carbonization of PVP during the annealing process, thus greatly enhancing the power factor.

To understand the effects of annealing on the TE performance of the optimized Te NWs thin pieces, the XRD patterns (Fig, S4, $\mathrm{ESI} \dagger$ ) and EDS spectrum for elemental analysis (Table S1, ESI $\dagger$ ) were obtained for these optimized Te NWs thin pieces with or without annealing. As observed in Fig. S4 (ESI $\dagger$ ), the Te NWs thin pieces after annealing showed the same diffraction peaks as hexagonal tellurium (JCPDS\# 36-1452). It should be noted that as the annealing temperature increased to $350{ }^{\circ} \mathrm{C}$, the intensity of the 100 and 110 peaks changed significantly and the 100 peak became much stronger. This indicates that the increase in annealing temperature led to a decrease in the diameter of Te NWs. ${ }^{59}$ Interestingly, the further increase in annealing temperature to 450 and $550{ }^{\circ} \mathrm{C}$ resulted in the intensity of the 100 peak being weakened again, suggesting the increase in the diameter of Te NWs. This revealed that the melting point of Te NWs was about $450{ }^{\circ} \mathrm{C}$. The further increase in the annealing temperature to $550{ }^{\circ} \mathrm{C}$ caused the re-aggregation of Te NWs and the larger diameter. Moreover, the crystallinity shown in Table S1 (ESI $\dagger$ ) changed with the increase in the annealing temperature. Increased crystallinity was observed for Te NWs thin pieces annealed at lower temperatures than $450{ }^{\circ} \mathrm{C}$. This increase in crystallinity would be beneficial for electrical conductivity. On the other hand, the $\mathrm{C}$ element content and the atom ratio of $\mathrm{C} / \mathrm{O}$ for these thin pieces annealed at temperatures lower than $450{ }^{\circ} \mathrm{C}$ were slightly changed, indicating no carbonization of glucose for these Te NWs thin pieces. $^{60}$ However, they were obviously increased when the annealing temperature was higher than $350{ }^{\circ} \mathrm{C}$, due to the carbonization of glucose and evaporation of Te atoms at high temperature. Therefore, as shown in Fig. 3E, the electrical conductivity of the optimized Te NWs thin pieces was decreased for a further increase in the annealing temperature, leading to the decreased TE performance at high temperature.

As mentioned above, the biomolecule glucose not only acts as a reductant, but can also act as a surfactant to assist in synthesizing Te NWs with promising electrical conductivity as compared to that synthesized with $\mathrm{N}_{2} \mathrm{H}_{4} \cdot \mathrm{H}_{2} \mathrm{O}$. Based on the surfactant role of PVP and glucose, a schematic illustration is proposed in Fig. 4A to understand the high electrical conductivity of the glucose-synthesized Te NWs. Since PVP is a polymer with strong polarity and a long chain, it can strongly adsorb on the surface of Te NWs to induce the nucleation and growth of Te NWs during the hydrothermal process, resulting in PVP-wrapped Te NWs. Simultaneously, some carbonaceous molecules resulting from the carbonization of PVP will also be adsorbed on the surface of Te NWs. However, the adsorption of PVP and carbonaceous molecules on the surface of Te NWs would critically block the contacts between Te NWs, which hinders the hopping of carriers among Te NWs. In addition, the presence of PVP and carbonaceous molecules may enhance the hopping barrier energy for carriers. Therefore, carriers with low energy would be greatly scattered at the interface between Te NWs, thus causing the inferior electrical conductivity of Te NWs. In contrast, as stated in Fig. 4A and Fig. S5 (ESI $\dagger$ ), there are only small carbonaceous molecules adsorbed on the surface of the glucose-synthesized Te NWs. Carriers can easily transport to the nearby Te NWs, resulting the high electrical conductivity of the glucose-synthesized Te NWs. In addition, as shown in Fig. 1, a pressing process was employed to fabricate the Te NWs thin pieces. The pressing process can result in a dense thin piece, which enhances the contact between Te NWs and enables the high electrical conductivity. 
(A)
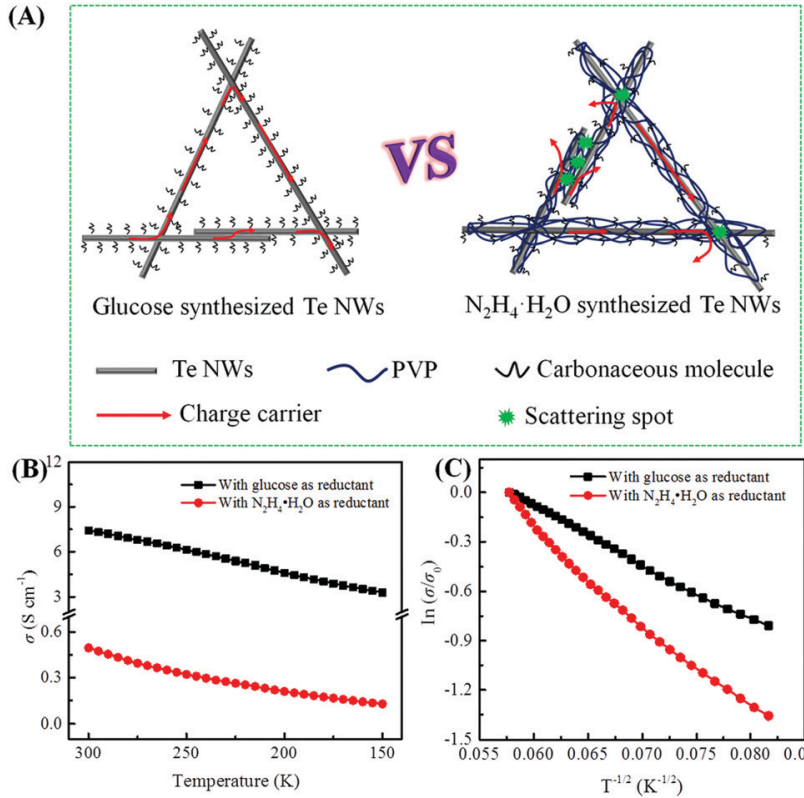

PVP

$\checkmark$ Carbonaceous molecule Scattering spot

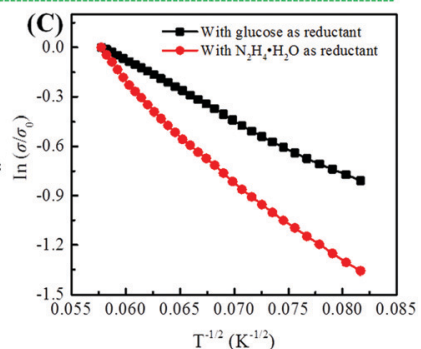

Fig. 4 Schematic illustration of the highly conductive Te NWs synthesized with glucose (A). The dependence of electrical conductivity on temperature (B) and analysis of the temperature-dependence of the electrical conductivity with the 1D VRH model (C) of the optimized Te NWs thin piece and that synthesized with $\mathrm{N}_{2} \mathrm{H}_{4} \cdot \mathrm{H}_{2} \mathrm{O}$ before annealing.

To illustrate the conductive properties and describe the high electrical conductivity of the glucose-synthesized Te NWs, the temperature-dependent electrical conductivity of the optimized Te NWs thin pieces and the $\mathrm{N}_{2} \mathrm{H}_{4} \cdot \mathrm{H}_{2} \mathrm{O}$-synthesized Te NWs before (Fig. 4B) and after (Fig. S6A, ESI $\dagger$ ) annealing at $350{ }^{\circ} \mathrm{C}$ for $3 \mathrm{~h}$ are presented. As seen in Fig. 4B, the electrical conductivity of the Te NWs thin pieces decreased with decreasing temperature for both glucose and $\mathrm{N}_{2} \mathrm{H}_{4} \cdot \mathrm{H}_{2} \mathrm{O}$-synthesized Te NWs, indicating typical semiconductor behavior. ${ }^{58}$ To reveal the high electrical conductivity of Te NWs synthesized with glucose, we fitted the temperature-dependent conductivity of these Te NWs thin pieces before and after annealing with the $1 \mathrm{D}$ variable range hopping $(\mathrm{VRH})$ model $\sigma(T)=\sigma_{0} \exp \left[\left(\frac{T_{0}}{T}\right)^{\frac{1}{1+d}}\right]$ in Fig. 4C and Fig. S6B (ESI $\dagger$ ). In the above formula, $\sigma_{0}$ and $T_{0}$ are the constants, the characteristic temperature related to the energy barrier constant for the hopping of carriers from nanowire to nanowire, and $d$ is the dimensionality of the conducting material (herein, the $d$ is 1$).{ }^{56}$ In this work, the estimated $T_{0}$ values for the Te NWs synthesized with glucose and $\mathrm{N}_{2} \mathrm{H}_{4} \cdot \mathrm{H}_{2} \mathrm{O}$ before annealing are 35 and $56 \mathrm{~K}$, respectively. The much lower $T_{0}$ for glucose-synthesized Te NWs can be used to illustrate the PVP surfactant-hindered transport properties, where the hopping of carriers was blocked and the high hopping barrier energy for carriers due to the strong wrapping of PVP on the surface of Te NWs. Therefore, the electrical conductivity of the glucose-synthesized Te NWs was significantly higher than that synthesized with $\mathrm{N}_{2} \mathrm{H}_{4} \cdot \mathrm{H}_{2} \mathrm{O}$. Furthermore, after annealing at $350{ }^{\circ} \mathrm{C}$ for $3 \mathrm{~h}$, not only was the $T_{0}$ value greatly
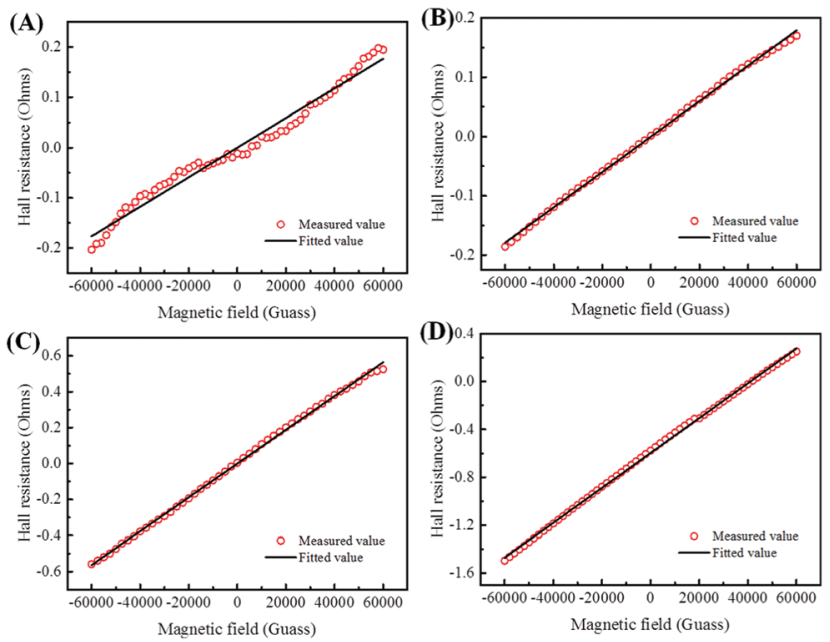

Fig. 5 The Hall resistance value as a function of magnetic field for the optimized Te NWs thin piece (A and C) and that synthesized with $\mathrm{N}_{2} \mathrm{H}_{4} \cdot \mathrm{H}_{2} \mathrm{O}$ $\left(B\right.$ and $D$ ) before ( $A$ and $B$ ) and after ( $C$ and $D$ ) annealing at $350{ }^{\circ} \mathrm{C}$ for $3 \mathrm{~h}$.

decreased to $20 \mathrm{~K}$, but also the electrical conductivity was dramatically enhanced to $7.44 \mathrm{~S} \mathrm{~cm}^{-1}$ for $\mathrm{N}_{2} \mathrm{H}_{4} \cdot \mathrm{H}_{2} \mathrm{O}$-synthesized Te NWs, which may be attributed to the degradation and carbonization of PVP during the annealing process. This can be further used to understand the hindered carriers by PVP and the high electrical conductivity of glucose-synthesized Te NWs. To fully reveal the high electrical conductivity of the glucose-synthesized Te NWs, we studied the transport parameters of both carrier concentration $(n)$ and mobility $(\mu)$ of the optimized Te NWs thin pieces and $\mathrm{N}_{2} \mathrm{H}_{4} \cdot \mathrm{H}_{2} \mathrm{O}$-synthesized Te NWs before and after annealing at $350{ }^{\circ} \mathrm{C}$ for $3 \mathrm{~h}$. Fig. 5 shows the Hall resistance value of these Te NWs thin pieces as a function of the magnetic field. According to the relationship between the Hall resistance and magnetic field, the $n$ and $\mu$ of these Te NWs thin pieces can be obtained. As listed in Table 1, both the $n$ and $\mu$ value of the glucose-synthesized Te NWs are much higher than that of the $\mathrm{N}_{2} \mathrm{H}_{4} \cdot \mathrm{H}_{2} \mathrm{O}$-synthesized Te NWs or previously reported NWs, suggesting that the presence of PVP on the surface of Te NWs may obstruct and scatter the carriers with low energy. Therefore, the glucose-synthesized Te NWs can present much higher electrical conductivity due to the larger $n$ and $\mu$ based on $\sigma=\mu n e$. Apart from investigating the electrical conductivity of the optimized Te NWs thin pieces with or without annealing at $350{ }^{\circ} \mathrm{C}$ for $3 \mathrm{~h}$ at low temperature, the temperature-dependent Seebeck coefficient of these Te NWs thin pieces was also studied as shown in Fig. S7 (ESI $\dagger$ ). A decreased $S$ value was observed for these Te NWs thin pieces with or without annealing. Therefore, decreased TE performance was observed in low-temperature conditions.

To assess the output performance of the optimized Te NWs thin pieces, we assembled a TEG prototype with 10 pieces of the optimized Te NWs thin pieces $\left(13.0 \times 2.0 \times 0.052 \mathrm{~mm}^{3}\right)$ as TE legs. As displayed in Fig. 6A, all the TE legs were connected by copper wire in series. The output voltage of the TEG versus the temperature difference was recorded as shown in Fig. 6B (black line). The output voltage was evenly increased with an increasing temperature difference $(\Delta T)$ from 0 to $80 \mathrm{~K}$. The slope of the 

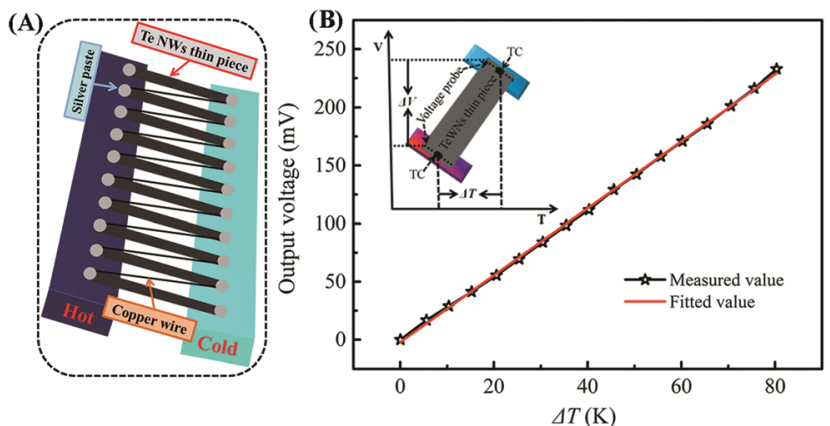

Fig. 6 The as-assembled TEG prototype with 10 pieces of the optimized Te NWs thin pieces (A). The output voltage of the TEG versus the temperature difference (B). The inset is the schematic illustration for measuring the $S$ value.

fitted line (red line in Fig. 6B) between the output voltage and $\Delta T$ was $2881 \mu \mathrm{V} \mathrm{K}^{-1}$, which is similar to the total $S_{\mathrm{tol}}$ value of the asassembled TEG based on the formula: $V_{\text {tol }}=n \times\left(S_{\mathrm{p}}-S_{\mathrm{n}}\right) \times \Delta T$, where $n, S_{\mathrm{p}}, S_{\mathrm{n}}$ are, respectively, the number of the p or n-type TE leg, and the Seebeck coefficients of the p and n-type TE legs. In our work, no n-type TE legs were employed; therefore, the value of $\mathrm{n}$ is 10 , and the $S_{\mathrm{p}}$ of the optimized Te NWs thin piece was measured as $282.5 \mu \mathrm{V} \mathrm{K}^{-1}$ above. The inset in Fig. $6 \mathrm{~B}$ is the schematic illustration for the measurement of the $S$ value. The output voltage can be up to $111 \mathrm{mV}$ at $\Delta T$ of $40 \mathrm{~K}$, and this value would be further improved to $231 \mathrm{mV}$ with $\Delta T$ of $80 \mathrm{~K}$. The output performance of our assembled TEG is one order of magnitude higher than that of the Te NMs-based TEG, even though no n-type TE legs were employed in this work, ${ }^{16,57,61,62}$ which is more promising for energy harvesting.

Finally, we investigated the air-stable TE performance of the optimized Te NWs thin pieces versus the time in ambient conditions, which are presented in Fig. S8 (ESI $\dagger$ ). As displayed, the electrical conductivity, Seebeck coefficient or power factor of the optimized Te NWs thin pieces synthesized with glucose or $\mathrm{N}_{2} \mathrm{H}_{4} \cdot \mathrm{H}_{2} \mathrm{O}$ degenerated with the extended time. For the Te NWs synthesized with glucose, the power factor was only decreased by $2 \%$ as compared with the original value when the fresh Te NWs thin pieces were kept at ambient conditions for 5 days, and it continuously decreased to $45 \%$ over 30 days. However, the power factor of the $\mathrm{N}_{2} \mathrm{H}_{4} \cdot \mathrm{H}_{2} \mathrm{O}$-synthesized Te NWs was decreased from 80 to $28 \%$ when the time was extended from 5 to 30 days. The significantly decreased power factor for the $\mathrm{N}_{2} \mathrm{H}_{4} \cdot \mathrm{H}_{2} \mathrm{O}$-synthesized Te NWs suggests that the glucosesynthesized Te NWs presented a more air-stable TE performance. To verify the more air-stable TE performance of the glucose-synthesized Te NWs, the C1s (Fig. S5, ESI $\dagger$ ) and Te3d (Fig. 2 and Fig. S9, ESI $\dagger$ ) XPS spectra of the Te NWs synthesized with glucose and $\mathrm{N}_{2} \mathrm{H}_{4} \cdot \mathrm{H}_{2} \mathrm{O}$ (both the fresh NWs and those stored under ambient conditions for 30 days) were recorded. As observed in the Te3d XPS spectra, the Te $3 \mathrm{~d}_{5 / 2}\left(\mathrm{Te}^{4+}\right)$ and Te $3 \mathrm{~d}_{3 / 2}$ $\left(\mathrm{Te}^{0}\right)$ peaks were present in all Te NWs samples. Notably, the integral ratio of $\mathrm{Te}^{3} \mathrm{~d}_{5 / 2}\left(\mathrm{Te}^{4+}\right)$ to $\mathrm{Te} 3 \mathrm{~d}_{3 / 2}\left(\mathrm{Te}^{0}\right)$ was increased with extending the time for both glucose and $\mathrm{N}_{2} \mathrm{H}_{4} \cdot \mathrm{H}_{2} \mathrm{O}$-synthesized Te NWs, but the increased value for the $\mathrm{N}_{2} \mathrm{H}_{4} \cdot \mathrm{H}_{2} \mathrm{O}$-synthesized
Te NWs was dramatically higher than that of the glucosesynthesized one, suggesting that the $\mathrm{N}_{2} \mathrm{H}_{4} \cdot \mathrm{H}_{2} \mathrm{O}$ synthesized Te NWs are easier to oxidize under ambient conditions. ${ }^{48}$ In addition, as seen in Fig. S5 (ESI $\dagger$ ), a stronger and wider C1s peak was observed for the glucose-synthesized Te NWs, illustrating that the presence of carbonaceous molecules on the surface of $\mathrm{Te}$ NWs would more effectively prevent Te NWs from being oxidized. Therefore, the glucose-synthesized Te NWs are more stable under ambient conditions and demonstrate the more air-stable TE performance. ${ }^{63}$

\section{Conclusion}

In conclusion, we synthesized highly conductive Te NWs via an eco-friendly hydrothermal process by employing the glucose biomolecule as a reductant. In this work, the Te NWs could be synthesized even when the reaction temperature was as low as $60{ }^{\circ} \mathrm{C}$. Compared with other wet chemistry methods, no harsh reductant and additional surfactants were employed in our work. On the one hand, we fabricated Te NWs thin pieces with the as-synthesized Te NWs to investigate their TE performance. By optimizing the reaction conditions, a high electrical conductivity of $8.44 \mathrm{~S} \mathrm{~cm}^{-1}$ and a large power factor of $67.5 \mu \mathrm{W} \mathrm{m} \mathrm{m}^{-1} \mathrm{~K}^{-2}$ were realized. The power factor can be further improved to $97.3 \mu \mathrm{W} \mathrm{m}{ }^{-1} \mathrm{~K}^{2}$ by an annealing process. The high electrical conductivity of the glucose-synthesized Te NWs was illustrated by the variable range hopping (VRH) model and carrier transport parameters. On the other hand, we assembled a simple TEG with 10 pieces of the as-fabricated Te NWs thin pieces without utilizing n-type TE legs. A promising output voltage of $231 \mathrm{mV}$ was obtained with the as-assembled TEG at the temperature difference of $80 \mathrm{~K}$. Finally, a stable TE performance of the as-fabricated Te NWs thin piece was illustrated by investigating the time-dependence of the TE performance. This work develops an eco-friendly strategy for highly conductive Te NWs and demonstrates a feasible design for Te NWsbased TE materials with promising TE performance.

\section{Associated contents}

Contents of ESI, $\uparrow$ are as follows: the fabrication of Te NWs thin piece with $\mathrm{N}_{2} \mathrm{H}_{4} \cdot \mathrm{H}_{2} \mathrm{O}$ synthesized Te NWs; the XPS spectra and SEM image of Te NWs synthesized with $\mathrm{N}_{2} \mathrm{H}_{4} \cdot \mathrm{H}_{2} \mathrm{O}$; the XRD pattern of Te NWs synthesized with glucose at $180^{\circ} \mathrm{C}$ for $12 \mathrm{~h}$ and $\mathrm{N}_{2} \mathrm{H}_{4} \cdot \mathrm{H}_{2} \mathrm{O}$; the TE performance of the as-fabricated Te NWs thin piece with $\mathrm{N}_{2} \mathrm{H}_{4} \cdot \mathrm{H}_{2} \mathrm{O}$ synthesized Te NWs versus annealing conditions; XRD patterns of optimized Te NWs thin pieces with or without annealing at different temperature for $3 \mathrm{~h}$; the $\mathrm{C} 1 \mathrm{~s}$ XPS spectra of the glucose (black line) and $\mathrm{N}_{2} \mathrm{H}_{4} \cdot \mathrm{H}_{2} \mathrm{O}$ (red line) synthesized fresh Te NWs; the temperature-dependent electrical conductivity and the analysis of the temperature-dependent electrical conductivity with the 1D VRH model of the optimized Te NWs thin piece and $\mathrm{N}_{2} \mathrm{H}_{4} \cdot \mathrm{H}_{2} \mathrm{O}$ synthesized Te NWs after annealing at $350{ }^{\circ} \mathrm{C}$ for $3 \mathrm{~h}$; temperature-dependent Seebeck coefficient of the optimized Te NWs thin pieces with or without annealing at $350{ }^{\circ} \mathrm{C}$ for $3 \mathrm{~h}$; the dependence of electrical 
conductivity, Seebeck coefficient, and power factor of the asfabricated Te NWs thin piece on time (days); the content of $\mathrm{C}$, atom ratio of $\mathrm{C} / \mathrm{O}$ and crystallinity of optimized Te NWs thin pieces with or without annealing at different temperature for $3 \mathrm{~h}$.

\section{Conflicts of interest}

There are no conflicts to declare.

\section{Acknowledgements}

We gratefully acknowledge the financial support of the National Natural Science Foundation of China (51762018, 51572117 and 51863009), the Innovation Driven "5511" Project of Jiangxi Province (20165BCB18016), the Natural Science Foundation of Jiangxi Province (20181ACB20010), the Jiangxi Provincial Department of Education (GJJ190584 and GJJ190612), and the Open Fund of the State Key Laboratory of Luminescent Materials and Devices (South China University of Technology, 2019-skllmd-01).

\section{Notes and references}

1 W. Z. Wu, G. Qiu, Y. X. Wang, R. X. Wang and P. D. Ye, Chem. Soc. Rev., 2018, 47, 7203-7212.

2 T. Chivers and R. S. Laitinen, Chem. Soc. Rev., 2015, 44, 1725-1739.

3 B. N. Zhong, G. T. Fei, W. B. Fu, X. X. Gong, S. H. Xu, X. D. Gao and L. D. Zhang, CrystEngComm, 2017, 19, 2813-2820.

4 J. Peng, Y. Pan, Z. Yu, J. J. Wu, J. C. Wu, Y. Zhou, Y. Q. Guo, X. J. Wu, C. Z. Wu and Y. Xie, Angew. Chem., Int. Ed., 2018, 57, 13533-13537.

5 W. He, H. V. Ngoc, Y. T. Qian, J. S. Hwang, Y. P. Yan, H. Choi and D. J. Kang, Appl. Surf. Sci., 2017, 392, 1055-1061.

6 Z. H. Wang, L. L. Wang, J. R. Huang, H. Wang, L. Pan and X. W. Wei, J. Mater. Chem., 2010, 20, 2457-2463.

7 G. Qiu, S. Y. Huang, M. Segovia, P. K. Venuthurumilli, Y. X. Wang, W. Z. Wu, X. F. Xu and P. D. Ye, Nano Lett., 2019, 19, 1955-1962.

8 S. Sharma, N. Singh and U. Schwingenschlogl, ACS Appl. Energy Mater., 2018, 1, 1950-1954.

9 H. R. Yang, S. W. Finefrock, J. D. Caballero and Y. Wu, J. Am. Chem. Soc., 2014, 136, 10242-10245.

10 G. D. Moon, S. Ko, Y. N. Xia and U. Jeong, ACS Nano, 2010, 4, 2307-2319.

11 D. Zhang, Y. H. Wang and Y. Yang, Small, 2019, 15, 1805241.

12 C. C. Li, F. X. Jiang, C. C. Liu, P. P. Liu and J. K. Xu, Appl. Mater. Today, 2019, 15, 543-557.

13 K. X. Chen, M. S. Li, D. C. Mo and S. S. Lyu, Front. Energy, 2018, 12, 97-108.

14 Fitriani, R. Ovik, B. D. Long, M. C. Barma, M. Riaz, M. F. M. Sabri, S. M. Said and R. Saidur, Renewable Sustainable Energy Rev., 2016, 64, 635-659.
15 H. Park, W. Son, S. H. Lee, S. Kim, J. J. Lee, W. Cho, H. H. Choi and J. H. Kim, CrystEngComm, 2015, 17, 1092-1097.

16 C. C. Li, F. X. Jiang, C. C. Liu, W. F. Wang, X. J. Li, T. Z. Wang and J. K. Xu, Chem. Eng. J., 2017, 320, 201-210.

17 Z. He, Y. Yang, J. W. Liu and S. H. Yu, Chem. Soc. Rev., 2017, 46, 2732-2753.

18 S. J. Ran, T. S. Glen, B. Li, T. Y. Zheng, I. S. Choi and S. T. Boles, J. Phys. Chem. C, 2019, 123, 22578-22585.

19 H. So, J. Yoo, K. Ryu, M. Yang and K. J. Lee, Ceram. Int., 2019, 45, 7226-7231.

20 J. Wang, M. Fang, G. T. Fei, M. Liu, G. L. Shang and L. D. Zhang, J. Mater. Sci., 2016, 51, 7170-7178.

21 M. Amani, C. L. Tan, G. Zhang, C. S. Zhao, J. Bullock, X. H. Song, H. Kim, V. R. Shrestha, Y. Gao, K. B. Crozier, M. Scott and A. Javey, ACS Nano, 2018, 12, 7253-7263.

22 D. W. Wang, A. J. Yang, T. S. Lan, C. Y. Fan, J. B. Pan, Z. Liu, J. F. Chu, H. Yuan, X. H. Wang, M. Z. Rong and N. Koratkar, J. Mater. Chem. A, 2019, 7, 26326-26333.

23 G. C. Xi, Y. K. Liu, X. Q. Wang, X. Y. Liu, Y. Y. Peng and Y. T. Qian, Cryst. Growth Des., 2006, 6, 2567-2570.

24 G. C. Xi, Y. Y. Peng, W. C. Yu and Y. T. Qian, Cryst. Growth Des., 2005, 5, 325-328.

25 M. S. Kim, X. H. Ma, K. H. Cho, S. Y. Jeon, K. Hur and Y. M. Sung, Adv. Mater., 2018, 30, 1702701.

26 W. F. Wang, J. Liu, X. Li, Q. L. Jiang, J. K. Xu, C. Luo, P. P. Liu, R. R. Tan, Y. K. Du and F. X. Jiang, J. Nanopart. Res., 2019, 21, 31.

27 Z. H. Ge and G. S. Nolas, Mater. Lett., 2017, 194, 20-22.

28 Z. L. Li, S. Q. Zheng, Y. Z. Zhang, R. Y. Teng, T. Huang, C. F. Chen and G. W. Lu, J. Mater. Chem. A, 2013, 1, 15046-15052.

29 R. Hassan and S. Ibrahim, Inorg. Chem. Commun., 2019, 104, 178-185.

30 L. Yang, Z. G. Chen, G. Han, L. N. Cheng, H. Y. Xu and J. Zou, Cryst. Growth Des., 2013, 13, 4796-4802.

31 Y. Li, W. Y. Zhao, X. Mu, X. Liu, D. Q. He, W. T. Zhu and Q. J. Zhang, J. Electron. Mater., 2016, 45, 1661-1668.

32 Z. P. Liu, Z. K. Hu, J. B. Liang, S. Li, Y. Yang, S. Peng and Y. T. Qian, Langmuir, 2004, 20, 214-218.

33 Y. P. Zuo, T. T. Li, D. W. Rao, X. M. Lei, Q. Li, G. L. Zhu, R. F. Lu and H. Y. Han, J. Phys. Chem. C, 2016, 120, 12305-12312.

34 D. Park, H. Ju, T. Oh and J. Kim, RSC Adv., 2018, 8, 8739-8746.

35 X. P. Wu, Y. Q. Wang, S. M. Zhou, X. Y. Yuan, T. Gao, K. Wang, S. Y. Lou, Y. B. Liu and X. J. Shi, Cryst. Growth Des., 2013, 13, 136-142.

36 B. N. Zhong, G. T. Fei, W. B. Fu, X. X. Gong, X. D. Gao and L. D. Zhang, Phys. Chem. Chem. Phys., 2016, 18, 32691-32696.

37 K. P. Wang, X. Y. Zhang, I. M. Kislyakov, N. N. Dong, S. F. Zhang, G. Z. Wang, J. T. Fan, X. Zou, J. Du, Y. X. Leng, Q. Z. Zhao, K. Wu, J. P. Chen, S. M. Baesman, K. Y. Liao, S. Maharjan, H. Z. Zhang, L. Zhang, S. A. Curran, R. S. Oremland, W. J. Blau and J. Wang, Nat. Commun., 2019, 10, 3985. 
38 Z. W. Wang, Y. B. Bu, Y. H. Zhao, Z. T. Zhang, L. F. Liu and H. Zhou, Environ. Sci. Pollut. Res., 2018, 25, 20756-20768.

39 S. Panigrahi, S. Kundu, S. K. Ghosh, S. Nath and T. Pal, J. Nanopart. Res., 2004, 6, 411-414.

40 M. S. Jin, G. N. He, H. Zhang, J. Zeng, Z. X. Xie and Y. N. Xia, Angew. Chem., Int. Ed., 2011, 50, 10560-10564.

41 G. S. Cao, X. J. Zhang, L. Su and Y. Y. Ruan, J. Exp. Nanosci., 2011, 6, 121-126.

42 C. Y. Chen, B. H. R. Suryanto, C. Zhao, X. C. Jiang and A. B. Yu, Small, 2015, 11, 3557-3567.

43 Z. Y. Wu, H. W. Liang, B. C. Hu and S. H. Yu, Angew. Chem., Int. Ed., 2018, 57, 15646-15662.

44 J. L. Wang, J. W. Liu, B. Z. Lu, Y. R. Lu, J. Ge, Z. Y. Wu, Z. H. Wang, M. N. Arshad and S. H. Yu, Chem. - Eur. J., 2015, 21, 4935-4939.

45 L. Q. Xu, Y. W. Ding, G. C. Xi, W. Q. Zhang, Y. Y. Peng, W. C. Yu and Y. T. Qian, Chem. Lett., 2004, 33, 592-593.

46 W. F. Wang, C. C. Li, X. Li, Y. H. Jia, F. X. Jiang, C. C. Liu, R. R. Tan and J. K. Xu, Thin Solid Films, 2018, 654, 23-29.

47 Q. F. Chen, G. H. Liu, G. X. Chen, T. Mi and J. L. Tai, BioResources, 2017, 12, 608-621.

48 Z. He, M. Hassan, H. X. Ju, R. Wang, J. L. Wang, J. F. Chen, J. F. Zhu, J. W. Liu and S. H. Yu, Nano Res., 2018, 11, 3353-3361.

49 M. Kalamuei, M. Mousavi-Kamazani and M. SalavatiNiasari, J. Nanostruct., 2014, 4, 459-465.

50 M. Safdar, X. Y. Zhan, M. T. Niu, M. Mirza, Q. Zhao, Z. X. Wang, J. P. Zhang, L. F. Sun and J. He, Nanotechnology, 2013, 24, 185705.
51 H. S. Qian, S. H. Yu, J. Y. Gong, L. B. Luo and L. F. Fei, Langmuir, 2006, 22, 3830-3835.

52 G. H. Li, X. Z. Cui, C. Y. Tan and N. Lin, RSC Adv., 2014, 4, 954-958.

53 R. C. Jin, G. Chen, C. S. Yan, D. H. Chen, H. M. Xu and J. Pei, CrystEngComm, 2012, 14, 8547-8553.

54 R. C. Jin, J. S. Liu and G. H. Li, Cryst. Res. Technol., 2014, 49, 460-466.

55 J. Choi, K. Lee, C. R. Park and H. Kim, Carbon, 2015, 94, 577-584.

56 P. L. Sun, C. C. Li, J. K. Xu, Q. L. Jiang, W. F. Wang, J. Liu, F. Zhao, Y. B. Ding, J. Hou and F. X. Jiang, Sustainable Energy Fuels, 2018, 2, 2636-2643.

57 Y. Wang, S. M. Zhang and Y. Deng, J. Mater. Chem. A, 2016, 4, 3554-3559.

58 C. C. Li, P. L. Sun, C. C. Liu, J. K. Xu, T. Z. Wang, W. F. Wang, J. Hou and F. X. Jiang, J. Alloys Compd., 2017, 723, 642-648.

59 J. W. Liu, J. Xu, W. Hu, J. L. Yang and S. H. Yu, ChemNanoMat, 2016, 2, 167-170.

60 M. I. Abd-Elrahman and S. M. Ahmed, Int. J. Polym. Mater., 2009, 58, 322-335.

61 Q. F. Meng, Q. L. Jiang, K. F. Cai and L. D. Chen, Org. Electron., 2019, 64, 79-85.

62 E. J. Bae, Y. H. Kang, K. S. Jang and S. Y. Cho, Sci. Rep., 2016, 6, 18805.

63 W. J. Lan, S. H. Yu, H. S. Qian and Y. Wan, Langmuir, 2007, 23, 3409-3417. 